\title{
e-Migrinter
}

$7 \mid 2011$

Réflexions croisées sur les migrations en Afrique de l'Ouest

\section{Migrants dans la ville. Une étude socio- anthropologique des mobilités migrantes à Salamanque}

Thèse soutenue le 9 novembre 2010 par Gunhild Odden à l'Université de Poitiers

\section{Gunhild Odden}

\section{OpenEdition}

\section{Journals}

Édition électronique

URL : https://journals.openedition.org/e-migrinter/907

DOI : 10.4000/e-migrinter.907

ISSN : 1961-9685

Éditeur

UMR 7301 - Migrinter

Édition imprimée

Date de publication : 1 septembre 2011

Pagination : 82-86

ISSN : 1961-9685

Référence électronique

Gunhild Odden, « Migrants dans la ville. Une étude socio-anthropologique des mobilités migrantes à Salamanque », e-Migrinter [En ligne], 7 | 2011, mis en ligne le 11 septembre 2017, consulté le 20 mai 2021. URL : http://journals.openedition.org/e-migrinter/907 ; DOI : https://doi.org/10.4000/emigrinter.907 


\title{
Migrants dans la ville. Une étude socio-anthropologique des mobilités migrantes à Salamanque
}

\author{
Thèse soutenue le 9 novembre 2010 par Gunhild Odden \\ à l'Université de Poitiers
}

C ette thèse se propose d'étudier la mobilité des migrants et ses effets sur l'évolution de l'espace urbain à Salamanque, croisant une sociologie des parcours migratoires et une sociologie urbaine avec comme clé de lecture la notion de projet migratoire. L'hypothèse de départ est celle d'un projet migratoire qui se dessine et se redessine au gré des rencontres et des opportunités. Par une procédure méthodologique compréhensive, s'inscrivant dans le cadre de la socio-anthropologie, il s'agit de saisir la logique des parcours, c'est-à-dire par une approche longitudinale de reconstituer les successions temporelles de mobilité, d'événements, de situations, de projets, et de décrire et d'analyser les manières d'investir spatialement et temporairement - la ville. Dans les lignes qui suivent seront présentés les principaux résultats de la recherche.

\section{Une ville moyenne de faible présence migratoire}

Cette recherche a d'abord montré que le choix d'effectuer un travail d'enquête dans une ville qui n'est pas une ville où la densité des migrants est forte est d'un intérêt réel. Cela a déjà été souligné dans d'autres recherches. Julie Garnier (2006, 2008), dans ses travaux sur les commerçants africains en Poitou-Charentes, montre notamment que derrière une présence migratoire numériquement faible peut se cacher une diversité importante en termes d'origines nationales. Cette observation est également valable pour Salamanque où 99 nationalités sont recensées (MTAS, 2007). Une présence migratoire faible n'est donc pas synonyme d'absence de diversité. L'étude des mobilités des migrants dans un tel contexte n'est cependant pas, selon Garnier, exempte de difficultés : elles demeurent «majoritairement invisibles » en l'absence de «quartiers labellisés », de "centralités commerciales» ou de concentration spatiale migratoire importante. Mais comme l'auteur le souligne, «cela présente néanmoins l'avantage d'interroger les outils méthodologiques dont nous disposons pour observer les présences et les initiatives étrangères dans la ville. Étudier des initiatives migrantes moins visibles en partant des espaces fréquentés et des situations urbaines de coprésence permet de considérer la question de «l'hétérogénéité sociale et culturelle» (Garnier, 2008 : 94). À partir d'espaces et d'acteurs différents au sein de la ville, et non seulement les espaces et les acteurs commerciaux, cette thèse a ainsi cherché à décrire des situations de coprésence variées, permettant de rendre compte de cette hétérogénéité sociale et culturelle dont parle Garnier : rencontres entre migrants étudiants et migrants économiques, entre migrants circulaires et migrants sédentaires, entre migrants commerçants et clients espagnols. Un premier apport de cette thèse est alors celui d'avoir procédé par une entrée par la ville, plutôt que par celle d'un ou plusieurs groupe(s) ethnique(s) prédéfini(s), et, ainsi, d'avoir rendu compte de dynamiques de mobilités divergentes, correspondant à des logiques et à des stratégies multiples, et ce dans un contexte de faible présence migratoire encore peu étudié.

D'un point de vue méthodologique, la recherche a plus particulièrement montré l'intérêt de procéder par une immersion dans la durée. Celle-ci a permis de rendre compte de l'évolution de différents lieux de la ville, de la reformulation du projet des informateurs et d'accéder à des informations que 
l'on n'obtient pas lors d'une première rencontre. D'ailleurs, ce dernier terme est caractéristique de la démarche inspirée par celle de Tarrius, cette rencontre avec l'Autre. Par une expérience relationnelle de l'enquête de terrain, j’ai cherché à montrer l'intérêt de rendre compte des conditions de production du savoir anthropologique : ma propre expérience et mon propre statut ont joué un rôle dans le choix du sujet et dans la construction des données et ont, ainsi, été dévoilés.

\section{Lire le parcours}

Quant à la notion de projet migratoire, constituant un fil rouge dans la carrière des migrants, elle apparait, selon moi, d'un grand intérêt pour lire le parcours, dans le temps et dans l'espace. Cette notion ne permet cependant pas à elle seule une lecture satisfaisante ; elle doit être associée à d'autres notions. Ma Mung propose une réflexion en termes de «conditions extérieures » et de «dispositions intérieures». Les conditions extérieures désignent alors « l'environnement dans lequel se réalisent les migrations » (Ma Mung, 2009: 27). Ici, l'environnement a d'abord été appréhendé en termes de contexte national d'une part, et de contexte local d'autre part.

Le contexte national ici est avant tout marqué par un passage récent de pays d'émigration à pays d'immigration; une augmentation du nombre d'étrangers spectaculaire, faisant du pays l'un des premiers pays européens récepteurs de migrants; un besoin de main-d'œuvre et, ainsi, des opportunités réelles d'emploi; et, enfin, une politique migratoire relativement souple. Plus récemment, ce même contexte a été bouleversé par des politiques migratoires plus restrictives et un marché du travail saturé.

Le contexte local, quant à lui, est marqué par une faible présence étrangère et un marché du travail peu opportun. Pour les étudiants, c'est la renommée de l'Université, les caractéristiques du castillan salmantin et/ou l'existence de réseaux formels (échanges institués) ou informels (amis ou autres connaissances) qui peuvent expliquer le choix de venir étudier dans la ville. Pour les migrants, les liens forts sont également mis en avant lorsqu'il s'agit de dire sa présence dans la ville. Cette tendance n'est pas une spécificité propre à cette ville, mais s'inscrit dans une logique globale des mouvements migratoires. En revanche, son statut de ville frontière (proximité avec le Portugal), de ville d'accueil (ONG accueillant des jeunes demandeurs d'asile) ou de ville à l'image tranquille caractérise plus spécifiquement le contexte local et s'inscrit dans cet « environnement des migrations ».

La capacité de puiser dans un réseau, de le mobiliser ou de contribuer à le créer, relève des dispositions intérieures et se révèle fructueuse en termes de projets, qu'il s'agisse de la réalisation d'un projet déjà identifié - généralement fruit, lui aussi, d'une rencontre antérieure - ou de l'identification d'un nouveau projet. C'est dire que la rencontre et le projet vont de pair, l'un engage l'autre et vice-versa. C'est aussi dire que notre hypothèse de départ est confirmée. Ce résultat s'inscrit dans la logique d'autres recherches empiriques menées sur le sujet (Pian, 2008, Hily et Rinaudo 2002). Tout individu n'est cependant pas doté des mêmes capacités relationnelles permettant de s'inscrire dans cette logique de projet, ou des capacités à mobiliser les réseaux existants. Le savoir-être et le savoir-faire - les dispositions intérieures - se révèlent alors inégalitaires dans l'expérience migratoire.

\section{Investir la ville}

Pour certains, la ville de Salamanque représente une première installation alors que d'autres ont vécu une expérience migratoire ailleurs avant de venir à Salamanque. D'autres encore y reviennent, soit dans la logique d'une circulation (des allers-retours qui se répètent), soit dans la logique d'une réinstallation (on y revient après un séjour plus ou moins long dans une 
autre ville espagnole ou dans un autre pays). Enfin, il y a ceux qui projettent de quitter la ville, dans un futur proche ou lointain. C'est dire que les parcours des migrants rencontrés sont marqués par des va-et-vient, des renouvellements de mouvements, des investissements dans différents lieux et des projections de mobilité future. Des parcours qui, à leur tour, se réalisent en fonction d'un projet en constante évolution, se dessinant et se redessinant en fonction des rencontres et des opportunités ; une logique m'amenant à avancer l'hypothèse selon laquelle on circule pour mieux s'installer. Ces migrants sont donc mobiles, mais ils sont également ancrés - d'une manière ou d'une autre - dans la ville.

Les institutions de la ville, et plus particulièrement les ONG et l'Église, se présentent comme des ressources importantes pour les migrants. Leur «bonne utilisation », semble cependant dépendre d'un projet de départ: l'obtention des papiers ou d'un hébergement, l'apprentissage de l'espagnol, la recherche d'un travail... En revanche, en l'absence d'un projet défini, faire de ces structures formelles une ressource semble plus difficile. Une autre caractéristique des ONG est celle d'organiser, en collaboration avec les associations de migrants, des événements culturels, donnant une visibilité importante à la «multi-culturalité » dans la ville. Néanmoins, ces manifestations traduisent surtout une volonté des autorités - qui les subventionnent - et des organisations de transmettre l'image d'une coprésence paisible, d'une «intégration réussie ». Les fêtes culturelles des associations des migrants, organisées par des personnes ressources du groupe, rentreraient dans la même typologie: si elles sont appréciées par les migrants, et témoignent d'une sociabilité en termes d'identification à tel ou tel groupe, ces événements ne sont que très ponctuels et ne donnent qu'une image très partielle parfois fabriquée en fonction de la construction sociale de l'immigration idéale - des dynamiques migratoires dans la ville. Ce sont surtout les associations qui se sont organisées pour la distribution de nourriture (l'association des Boliviens), de bolsa de trabajo (système de recrutement) ou de microcrédits (l'association des LatinoAméricains) que l'on peut catégoriser comme jouant un rôle allant au-delà de la simple visibilité ethnique du groupe au sein de la ville.

Les parcs, le marché hebdomadaire et la scène nocturne quant à eux sont représentatifs des lieux de «l'ethnicisation » de la scène urbaine. Si les Boliviens se sont appropriés la Plaza de Burgos, le Parque de los Jesuitas est fréquenté par des groupes aux statuts et origines divers. Au marché, ce sont surtout les Sénégalais qui ont su se faire une place, tout en collaborant avec la population locale gitane, toujours majoritaire, et l'investissement se joue sous la forme d'apprentissage du rôle de vendeur, d'accord tacite, de tolérance, de stratégies, d'un ordre établi en fonction de l'ancienneté. L'étude de la scène nocturne nous montre comment l'on « apprend à faire la fête »; comment ce cadre pour beaucoup constitue un lieu propice pour nouer des liens, amicaux ou amoureux; comment les interactions de la nuit ne sont pas les mêmes que celles de la journée. L'étude du quartier Garrido a permis d'analyser les interactions avec les Espagnols; de montrer comment le migrant commerçant a su et pu s'insérer dans un contexte local marqué par une tradition ouvrière et comment cette insertion a mené à la création de nouvelles formes de sociabilités entre autochtones et migrants. Si la plupart des établissements développent une stratégie de proximité, d'autres attirent une clientèle venant de l'extérieur, donnant ainsi du sens au quartier à l'échelle de la ville. Quartier porteur de dynamiques contemporaines, on vient désormais à Garrido Norte pour chercher ce que l'on ne trouve pas ailleurs. Ces caractéristiques (quartier ouvrier qui devient un quartier de migrants, «mixité » de produits/services/clients, absence de phénomène de "ghettoïsation») ne sont cependant pas propres au quartier Garrido Norte ou à Salamanque, mais se 
retrouvent dans d'autres villes moyennes en Espagne, ce qui m'amène à décrire Salamanque comme un modèle de ville moyenne, mais aussi comme un modèle de ville espagnole moins receveuse de migrants.

Aujourd'hui à Salamanque, les migrants tentent donc d'organiser leurs quotidiennetés, leurs installations provisoires ou plus durables, dans un milieu urbain loin des capitales comme Madrid ou Barcelone. Sans être particulièrement caractérisée par des politiques généreuses pour intégrer les nouveaux arrivés, Salamanque offre cependant des possibilités, certes peu importantes, d'accéder à des ressources dans un contexte urbain où des rencontres et des échanges entre les établis et les nouveaux arrivés sont réels. Les ressources utiles de l'espace local sont donc accessibles sans trop de difficultés : le migrant sait vite comment se repérer et à quelle porte frapper dans la ville.

Situer mes enquêtes à Salamanque s'est avéré d'une grande pertinence pour analyser les modalités d'organisation des migrants et saisir la logique des parcours et des manières d'investir la ville. Ce type de configuration urbaine qualifiée de "ville moyenne » peut alors permettre, par contraste avec les dynamiques observées dans les métropoles, de reposer la question des places qu'occupe l'étranger lors de ses cheminements migratoires, soit une orientation ramenant finalement au statut de l'espace et à sa signification sociale.

Gunhild Odden Chercheure au Centre pour la communication interculturelle (SIK), Stavanger, Norvège gunhild.odden@sik.no
Bibliographie

Garnier, Julie (2008) Mettre en scène l'altérité : stratégies, enjeux et contraintes. Le point de vue des "commerçants africains" en milieu urbain, Diversité urbaine, Vol. 8, $\mathrm{N}^{\circ} 1$, pp. 89-112.

Garnier, Julie (2006) Pratiques de l'échange, circulations et stratégies spatio-temporelles: la mise en scène des commerçants africains en milieu urbain, Poitiers, Université de Poitiers, 582 p.

Th. Doct. : Socoi. : Poitiers : 2006.

Hily, Marie-Antoinette ; Rinaudo, Christian (2002a) Entrepreneurs migrants sur le marché de Vintimille, in Petaldi, Michel (Dir.), La fin des norias? Réseaux migrants dans les économies marchandes en Méditerranée, Paris, Maisonneuve \& Larose, pp. 335-351.

Ma Mung, Emmanuel (2009) Le point de vue de l'autonomie dans l'étude des migrations internationales: "penser de l'intérieur " les phénomènes de mobilité, in Dureau, Françoise ; Hily, Marie-Antoinette (Dir.), Les Mondes de la mobilité, Rennes, Presses Universitaires de Rennes, pp. 25-38.

Pian, Anaiik (2009) Aux frontières de l'Europe. L'aventure incertaine des Sénégalais au Maroc, Paris, La Dispute, 237 p. 